\title{
Embryonal Rhabdomyosarcoma of the Ampulla of Vater With Long-Term Survival Following Pancreaticoduodenectomy
}

\author{
By Michael G. Caty, Keith T. Oldham, and Edward V. Prochownik \\ Ann Arbor, Michigan
}

\begin{abstract}
Rhabdomyosarcoma of the biliary tree is a rare cause of biliary tract obstruction in childhood. A 3-year-old child is reported here after presenting with obstructive jeundice secondary to an embryonal rhabdomyosarcoma of the ampulla of Vater. He underwent pancreaticoduodenectomy followed by adjuvant chemotherapy and irradiation. $\mathrm{He}$ is now well and free of disease 5 years following treatment. This child appears to be the first long-term survivor who has required pancreaticoduodenal resection for this lesion. (a) 1990 by W.B. Saunders Company.
\end{abstract}

INDEX WORDS: Rhabdomyosarcoma; biliary rhabdomyosarcoma.

$\mathbf{R}$ HABDOMYOSARCOMA of the biliary tree, although rare, is the most common cause of neoplastic biliary obstruction in childhood. ${ }^{1}$ Prior to the uniform use of multimodal therapy to treat this neoplasm, survival was uncommon. A review by Lack et al of all cases reported prior to 1979 showed only four survivors of 23 patients, with the average time from onset of symptoms to death being 6.3 months. ${ }^{2}$ Poor survival from this tumor has been attributed to its strategic anatomical location, a propensity for liver invasion, and frequent delay in diagnosis.

One variant of this tumor is embryonal rhabdomyosarcoma of the ampulla of Vater. In 1978, Isaacson ${ }^{3}$ reported the first case of this specific histology and location in $61 / 2$-year-old child in whom the diagnosis was made at autopsy. Herein, we present a patient, we believe to be the first, with a rhabdomyosarcoma of the ampulla of Vater in whom long-term survival has been achieved with pancreaticoduodenectomy combined with chemotherapy and radiotherapy.

\section{CASE REPORT}

An otherwise healthy, 3-year-old white boy presented in August 1985 with poorly localized abdominal pain, anorexia, and fever initially thought to be gastroenteritis. Two weeks later he was admitted to the hospital for persistent and increasing pain, nausea, vomiting, jaundice, and pruritis, with a presumptive diagnosis of

From the Section of Pediatric Surgery. Department of Surgery. and the Section of Hematology-Oncology, Department of Pediatrics, University of Michigan Medical Center, Ann Arbor, MI.

Address reprint requests to Keith T. Oldham, MD. University of Michigan/Pediatric Surgery, 7516 Mott Children's Hospital, Box 0245, Ann Arbor, MI 48109-0245.

(C) 1990 by W.B. Saunders Company.

0022-3468/90/2512-0019\$03.00/0 hepatitis. On physical examination the patient was icteric with mild but nonspecific abdominal tenderness and slight hepatomegaly. His white blood cell count was $13,000 / \mathrm{mm}^{3}$ with a normal differential count and his hematocrit was $29.8 \%$. His liver function tests were as follows: aspartate aminotransferase $345 \mathrm{IU} / \mathrm{L}$ (normal, 2 to 35 ), alkaline phosphatase 1,256 IU/L (normal, 70 to 350 ), and total bilirubin $8.2 \mathrm{mg} / \mathrm{dL}$ (direct, $4.0 \mathrm{mg} / \mathrm{dL}$ ). An ultrasound examination was remarkable for intrahepatic and extrahepatic biliary tract dilation and a predominantly cystic mass with some solid component in the head of the pancreas. Computed tomography of the abdomen confirmed the presence of a cystic mass in the head of the pancreas, $2.1 \mathrm{~cm}$ in diameter (Fig 1). An upper gastrointestinal series with barium showed a dilated duodenal loop and inflammatory changes involving the duodenal mucosal. Preoperatively, a type III choledochal cyst was believed to be the most likely diagnosis.

The patient underwent exploratory laparotomy, at which a cystic mass was found within the posterior aspect of the head of the pancreas, contiguous with the distal common bile duct. An intraoperative cholangiogram showed flow into a cystic structure that was in continuity with the common bile duct (Fig 2). The cystic mass was opened and botryoid, gelatinous material was spontaneously extruded from the cyst cavity. This material and the wall of the cyst were sent for frozen section evaluation, showing a rhabdomyosarcoma. No metastatic disease was apparent and the tumor was judged to be surgically resectable. A pancreaticoduodenectomy was performed. Dissection along the portal vein established the absence of vascular invasion. The dilated common bile duct was transected just proximal to the cystic duct. The pancreas was transected just to the left of the portal vein. The gastric antrum and duodenum were completely resected. The gastrointestinal tract was reconstructed using a retrocolic jejunal limb to which an end-to-side choledochojejunostomy, end-to-side pancreaticojejunostomy, and gastrojejunostomy were established. The patient recovered uneventfully and was discharged home on the 10th postoperative day. Final pathology on

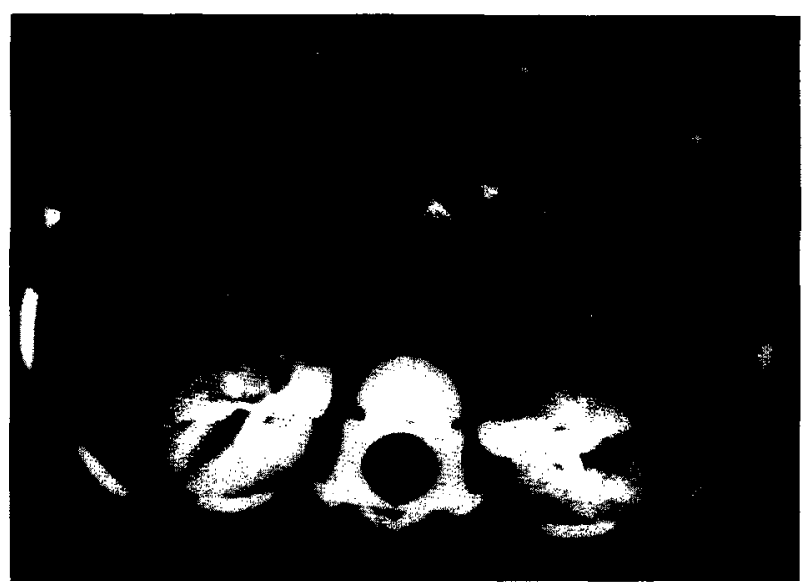

Fig 1. The preoperative computed tomography scan demonstrates a dilated gallbladder, intrahepatic biliary duct dilation, and a cystic mass within the head of the pancreas that is contiguous with the distal common bile duct (arrow). 


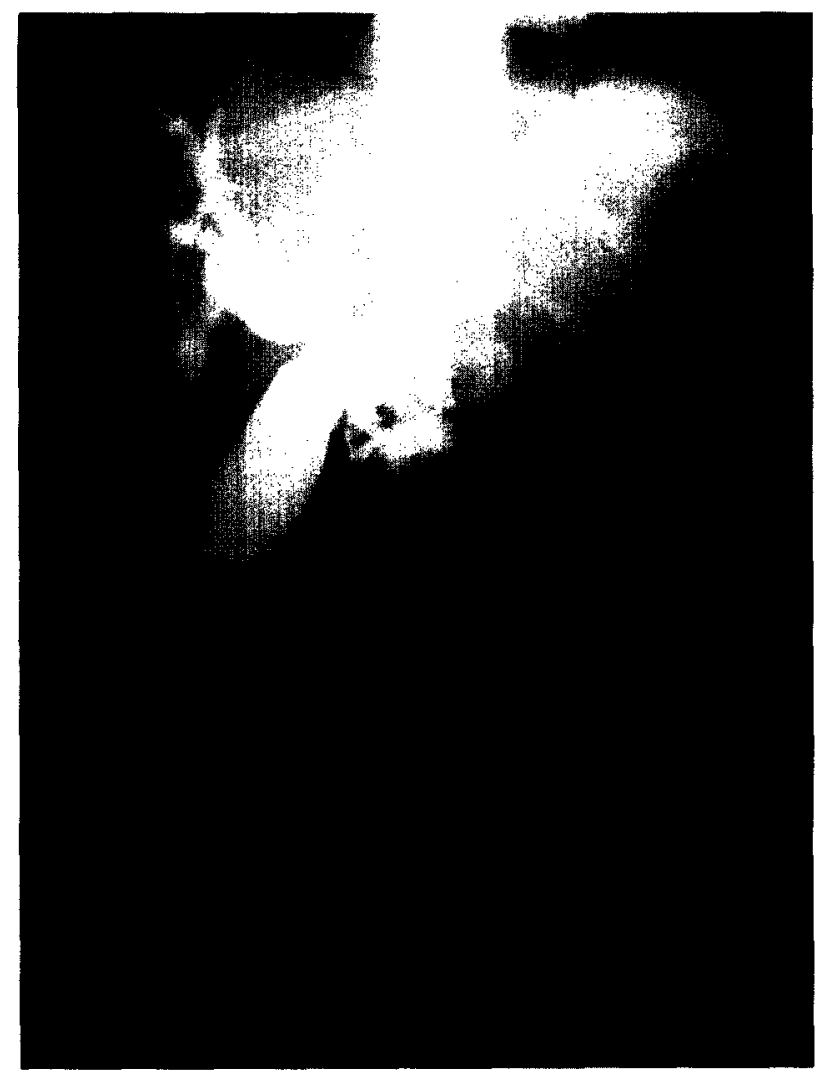

Fig 2. The intraoperative cholangiogram demonstrates intrahepatic and extrahepatic biliary duct dilation with the presence of an exophytic neoplasm (arrow) in the distal common bile duct.

the specimen confirmed an embryonal rhabdomyosarcoma arising from the ampulla of Vater. Microscopic residual tumor was found at the proximal bile duct margin; common duct lymph nodes were free of disease (Fig 3).

Postoperative evaluation including computed tomography of the chest and a bone scan demonstrated no evidence of metastatic disease. The patient was randomized to Arm 33 of the Stage III protocol of the Intergroup Rhabdomyosarcoma Study group. He received 1 year of adjuvant chemotherapy with Vincristine, Actinomycin D, and Adriamycin. His course was complicated by two hospitalizations for chemotherapy-related myelosuppression, which were unassociated with sepsis. The patient also received $3,420 \mathrm{rad}$ of external beam radiation to the field of the tumor bed and positive margin regions. This was given in 19 fractions over 25 days.

The patient's postoperative course was significant for radiationinduced gastritis and enteritis. This resolved while the patient was supported by total parenteral nutrition. After resuming enteral feeding the patient exhibited both pancreatic exocrine insufficiency and the dumping syndrome. These resolved with diet modification and pancreatic enzyme replacement. No evidence of pancreatic endocrine insufficiency developed. Currently the patient is 5 years after resection, free of disease, tolerating a near normal diet, and developing normally.

\section{DISCUSSION}

Rhabdomyosarcoma of the biliary tree is thought to be the most common neoplastic cause for biliary tract obstruction in children. However, it is rare; approximately one case is reported annually in the United States.

The signs and symptoms of this patient typify the presentation of rhabdomyosarcoma of the biliary tree in childhood. This child presented at the age of 3 years with obstructive jaundice. Preoperatively, an alternative diagnosis such as hepatitis or choledochocele cyst was considered. This child had gross disease confined to the head of the pancreas and was therefore amenable to resection. Late recognition of this malignancy and extension into the liver may preclude resection.

Despite the historic plea for aggressive surgery to treat rhabdomyosarcoma of the biliary tree, ${ }^{4,5}$ improvements in survival have only been achieved with multidisciplinary therapy. ${ }^{6}$ The addition of chemotherapy and radiation to surgical resection has increased survival for rhabdomyosarcoma of the biliary tree. A recent review by Ruymann of all patients with biliary rhabdomyosarcoma enrolled in the Intergroup Rhabdomyosarcoma Studies (I and II) showed an overall survival rate of $40 \%$ in a cohort of 10 patients. Survivors were limited to those children who had surgical resection of gross tumor followed by chemotherapy and/or radiotherapy. We believe this patient to be the solc longterm survivor of biliary rhabdomyosarcoma when the surgical resection required pancreaticoduodenectomy.

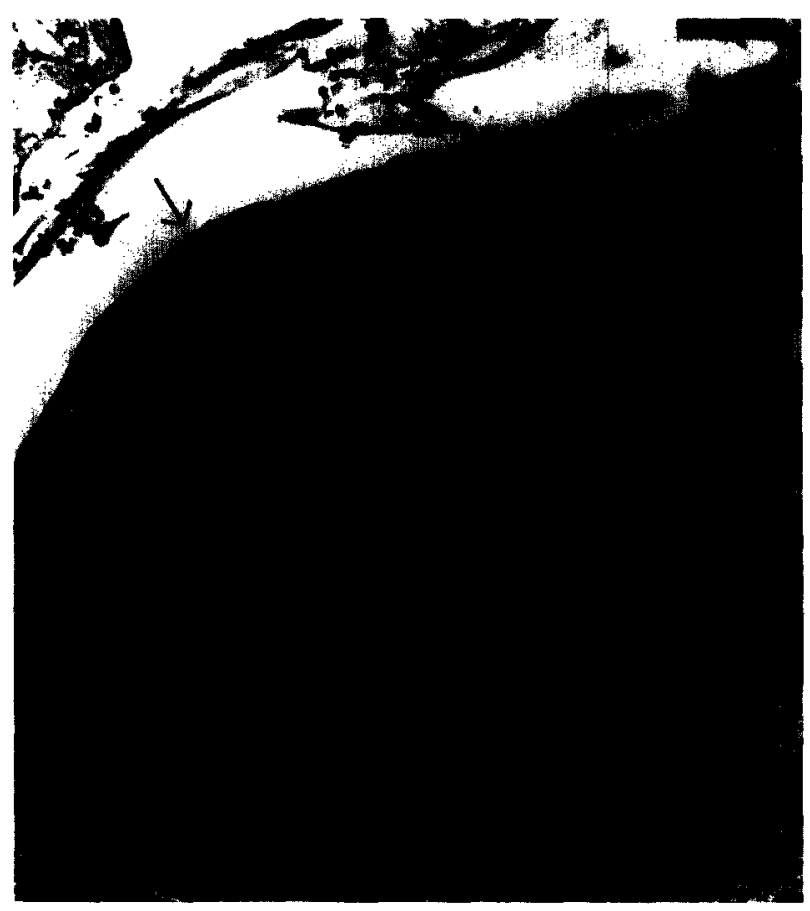

Fig 3. Permanent microscopic sections (original magnification $\times 140$ ) illustrate an embryonal rhabdomyosarcoma that appears to arise from the distal common bile duct (arrow). 


\section{REFERENCES}

1. Davis GI, Kissane JM, Ishak KG: Embryonal rhabdomyosarcoma (sarcoma boytryoides) of the biliary tree. Cancer 24:333-342, 1969

2. Lack EE, Perez-Atayde AR, Schuster SR: Botryoid rhabdomyosarcoma of the biliary tract. Am J Surg Pathol 5:643-652, 1981

3. Isaacson C: Embryonal rhabdomyosarcoma of the ampulla of Vater. Cancer 41:365-368, 1978
4. Akers RD, Needham ME: Sarcoma botryoides of the bile ducts with survival. J Pediatr Surg 6:474-479, 1971

5. Martinez-F LA, Haase GM, Koep LJ, et al: Rhabdomyosarcoma of the biliary tree: The case for aggressive surgery. J Pediatr Surg 17:508-511, 1982

6. Ruymann FB, Raney RB, Crist WM: Rhabdomyosarcoma of the biliary tree in childhood. Cancer 56:575-581, 1986 